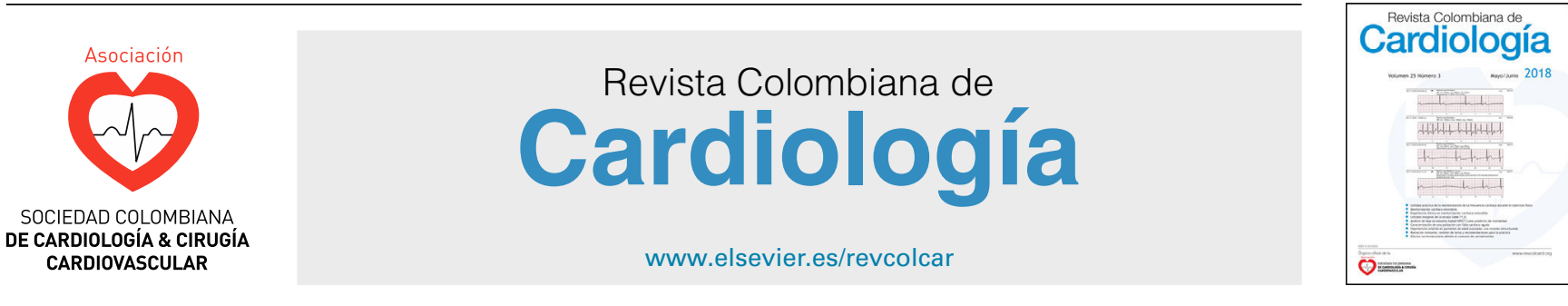

CARDIOLOGÍA DEL ADULTO - ARTÍCULO ORIGINAL

\title{
Sistemas dinámicos y teoría de la probabilidad aplicados al diagnóstico de la dinámica cardiaca en dieciséis horas
}

\author{
Javier Rodríguez ${ }^{\mathrm{a}, \mathrm{b}, \mathrm{c}, *}$, Signed Prieto ${ }^{\mathrm{a}, \mathrm{b}}$, Catalina Correa ${ }^{\mathrm{a}, \mathrm{b}}$, Sandra Medina ${ }^{\mathrm{a}, \mathrm{b}}$, \\ Sefirot Rodríguez ${ }^{\mathrm{a}, \mathrm{b}}$, Diana Margarita Cardona ${ }^{\mathrm{a}, \mathrm{b}}$, Nathalia López ${ }^{\mathrm{d}}$ y Fredy López ${ }^{\mathrm{a}, \mathrm{b}}$
}

\author{
a Grupo Insight, Bogotá, Colombia \\ b Centro de Investigaciones, Clínica del Country, Bogotá, Colombia \\ c Universidad Militar Nueva Granada, Bogotá, Colombia \\ d Universidad de Los Andes, Bogotá, Colombia
}

Recibido el 23 de febrero de 2017; aceptado el 2 de abril de 2019

Disponible en Internet el 13 de julio de 2019

\section{PALABRAS CLAVE \\ Frecuencia cardiaca; \\ Sistemas dinámicos; \\ Fractales; \\ Probabilidad; \\ Diagnóstico; \\ Dinámica cardiaca}

\begin{abstract}
Resumen
Introducción: se han establecido diagnósticos cuantitativos de los sistemas cardiacos, partiendo de teorías como los sistemas dinámicos, la geometría fractal y la teoría de probabilidad.

Objetivo: evaluar la dinámica cardiaca con base en una metodología fundamentada en la teoría de probabilidad y los sistemas dinámicos, en dieciséis horas.

Metodología: a partir de ochenta registros electrocardiográficos de dinámicas cardiacas, diez normales y setenta con enfermedad, se tomaron los valores máximos y mínimos de la frecuencia cardiaca y el número de latidos/hora durante cada hora, con los cuales se construyó el atractor. Posteriormente, se calculó la dimensión fractal por el método de box counting, los espacios de ocupación y la probabilidad de los espacios de ocupación del atractor. Se determinó el diagnóstico matemático y se hizo una validación estadística respecto al diagnóstico convencional, tomado como estándar de oro.

Resultados: se evidenció que la probabilidad de ocupación espacial de los atractores de dinámicas patológicas estuvo entre 0,029 y 0,144 y para dinámicas en estado de normalidad entre 0,164 y 0,329 . Se hallaron valores de sensibilidad, especificidad, valor predictivo positivo y negativo de $100 \%$ y coeficiente kappa de 1.

Conclusiones: se pudo confirmar la capacidad diagnóstica y predictiva de la metodología para diferenciar estados normales de patológicos a nivel clínico.

(c) 2019 Sociedad Colombiana de Cardiología y Cirugía Cardiovascular. Publicado por Elsevier España, S.L.U. Este es un artículo Open Access bajo la licencia CC BY-NC-ND (http:// creativecommons.org/licenses/by-nc-nd/4.0/).
\end{abstract}

\footnotetext{
* Autor para correspondencia.

Correo electrónico: grupoinsight2025@yahoo.es (J. Rodríguez).
} 


\section{KEYWORDS}

Heart rate;

Dynamic systems;

Fractals;

Probability;

Diagnosis;

Cardiac dynamics
Dynamic systems and probability theory applied to the diagnosis of cardiac dynamics in 16 hours

\begin{abstract}
Introduction: Quantitative diagnostics of cardiac systems have been established using theories such as, dynamic systems, fractal geometry, and probability theory.

Objective: To evaluate cardiac dynamics using a methodology based on probability theory and dynamic systems in sixteen hours.

Methods: Using a total of 80 cardiac dynamic electrocardiograph traces (10 normal and 70 with disease), a record was made of the maximum and minimum heart rate values, as well as the number of heart beats/hour during each hour. These values were used to construct the attractor. The fractal dimension was then calculated using the "box counting" method, the spatial occupation, and the probability of spatial occupation by the attractor. The mathematic diagnosis was determined, and a statistical validation was made as regards the conventional diagnosis, which was taken as the reference standard.

Results: It was shown that the probability of spatial occupation of the pathological attractor dynamics was between 0.29 and 0.144 , and for dynamics in the normal state it was between 0.164 and 0.329 . The sensitivity, specificity, positive and negative predictive values were $100 \%$, and the kappa coefficient was 1.

Conclusions: The diagnostic and predictive capacity of the methodology to differentiate normal from disease states at clinical level was demonstrated.

() 2019 Sociedad Colombiana de Cardiología y Cirugía Cardiovascular. Published by Elsevier España, S.L.U. This is an open access article under the CC BY-NC-ND license (http:// creativecommons.org/licenses/by-nc-nd/4.0/).
\end{abstract}

\section{Introducción}

El objetivo de la teoría de los sistemas dinámicos es estudiar el estado y la evolución de los sistemas, lo cual se logra mediante la representación gráfica de las variables dinámicas en el denominado espacio de fases, originando estructuras geométricas conocidas como atractores. Existen tres tipos de atractores: cíclicos, puntuales y caóticos ${ }^{1}$. La dimensión fractal es la medida con la cual se observa la irregularidad del atractor caótico ${ }^{2}$. Por otro lado, la medida de las posibilidades de que ocurra un evento ha sido determinada por la teoría de la probabilidad, pues es por medio de esta que se cuantifica el acaecimiento o no de un hecho, asignándole valor entre 0 y $1^{3,4}$. Desde conceptos de la Física y la Matemática como los mencionados, se han logrado establecer diagnósticos de la dinámica cardíaca fetal y del adulto $^{4-6}$.

En el mundo, la principal causa de mortalidad corresponde a las enfermedades cardiovasculares (ECV); las formas isquémicas como el infarto agudo de miocardio (IAM) son las más relacionadas con letalidad ${ }^{7}$. De acuerdo con la Organización Mundial de la Salud, el 1,9\% de las muertes al año en el continente americano obedecen a las ECV; también, se ha estimado que una de cada cuatro personas padece este tipo de enfermedad, y se proyecta que para el año 2030 pueden llegar a morir 23.6 millones de personas por esta condición ${ }^{8}$.

El dispositivo Holter fue creado con el fin de diagnosticar y hacer seguimiento ambulatorio a los pacientes; permite detectar alteraciones que no son evidenciables en el electrocardiograma convencional. De otro lado, el monitor electrocardiográfico continuo se usa en la Unidad de
Cuidados Intensivos para evaluar la dinámica cardiaca de pacientes en estado crítico ${ }^{7}$. Por medio de estas herramientas es posible observar alteraciones del ritmo cardiaco y la eficacia de los métodos terapéuticos.

El comportamiento de la dinámica cardiaca de un sistema que varía en el tiempo, es susceptible de estudio a partir de la teoría de los sistemas dinámicos; en este sentido, se han diseñado diferentes metodologías para su evaluación ${ }^{9}$. En diferentes estudios, se ha buscado establecer la variabilidad de la frecuencia cardiaca (VFC) como parámetro diferenciador para realizar diagnósticos, utilizando conceptos provenientes de las matemáticas, como la teoría de los sistemas dinámicos y la geometría fractal ${ }^{10}$. Goldberger et al. establecieron que las dinámicas cardiacas que presentan un alto grado de regularidad o que son extremamente aleatorias, suelen asociarse a enfermedades, mientras que el estado de normalidad se halla en un punto medio entre estos extremos ${ }^{11}$. De igual manera, se han encontrado métodos que permiten predecir la mortalidad en pacientes con infarto de miocardio y fracción de eyección al $35 \%$. Sin embargo, la aplicabilidad clínica de estos parámetros continúa en estudio ${ }^{12}$.

Con base en los sistemas dinámicos y la geometría fractal $^{13}$, se han desarrollado metodologías de carácter diagnóstico que logran diferenciar de forma objetiva y reproducible enfermedad de normalidad ${ }^{5}$. Por ejemplo, se desarrolló un método diagnóstico fundamentado en la evaluación de los espacios de ocupación de los atractores caóticos cardiacos en el espacio fractal de box-counting. Este método fue confirmado en estudios de evaluación de su aplicabilidad clínica ${ }^{14}$, incluso ante una disminución de su tiempo de evaluación a dieciséis horas ${ }^{15}$. 
La teoría de la probabilidad aplicada a la frecuencia cardiaca y el número de latidos/hora, ha permitido un método de evaluación objetivo y reproducible que establece diferenciaciones entre enfermedad y normalidad, cuya efectividad se ha confirmado en diferentes estudios, alcanzando niveles de sensibilidad y especificidad de $100 \%$ y un coeficiente kappa de $1^{16}$.

Recientemente se desarrolló una metodología que unifica la teoría de sistemas dinámicos, la geometría fractal y la teoría de la probabilidad, logrando un diagnóstico de la dinámica cardiaca que evalúa la probabilidad de ocupación espacial de atractores cardiacos en el espacio fractal de box counting. Así se diagnostica efectivamente normalidad de enfermedad, con información de la frecuencia cardiaca y el número de latidos durante dieciséis horas (en evaluación para publicación). Este trabajo tiene como propósito llevar a cabo una confirmación de la aplicabilidad clínica de esta metodología diagnóstica, por medio de un estudio ciego con ochenta estudios Holter y registros electrocardiográficos continuos.

\section{Materiales y métodos}

\section{Definiciones}

Espacio de fases. Es un espacio geométrico n-dimensional para $n>1$, en el cual es posible representar la dinámica de los sistemas, por medio de la graficación de pares ordenados consecutivos en el tiempo de una misma variable dinámica. Esta representación se denomina atractor.

Fractal. Es un objeto cuya irregularidad y dimensión puede ser calculada con diferentes métodos. Para fractales de tipo salvaje, el método usual es el método de boxcounting.

Dimensión fractal de box-counting. Se mide por medio de la siguiente ecuación:

$D_{F}=-\frac{\log N_{1}\left(2^{-(j+1)}\right)-\log N_{2}\left(2^{-j}\right)}{\log 2^{j+1}-\log 2^{j}}=\log 2-\frac{N_{1}\left(2^{-(j+1)}\right)}{N_{2}\left(2^{-j}\right)}$

Donce $D_{F}$ es la dimensión fractal, $\mathrm{N}$ es el número de cuadros ocupados por el objeto y $\mathrm{j}$ es el grado de partición de la cuadrícula.

Probabilidad de ocupación del atractor POA. Se define como el cociente entre el número de cuadros ocupados por el atractor cardiaco y el número total de cuadros del espacio generalizado de box-counting.

$\mathrm{POA}=\frac{\text { Nüméro de cuadro ocupados por el atractor }}{\text { Total de cuadros del espacio }}$

\section{Población}

Se seleccionaron para el estudio, ochenta registros electrocardiográficos ambulatorios y continuos durante dieciséis horas, correspondientes a pacientes mayores de veintiún años, entre los cuales diez fueron diagnosticados por un cardiólogo experto como normales y los restantes setenta como enfermos. Estos registros provenían de bases de datos del grupo Insight. El grupo de diez registros Holter normales fue seleccionado como control, porque no presentaba dudas en su diagnóstico clínico, con el fin de comparar estos resultados con los registros Holter anormales y establecer diferencias matemáticas claras entre los distintos estados evaluados.

\section{Procedimiento}

Fueron enmascarados los diagnósticos convencionales, establecidos por personal experto. Posteriormente, se tomaron el número de latidos/hora y las frecuencias máximas y mínimas de cada registro Holter y/o electrocardiográfico continuo, para generar una secuencia semialeatoria de frecuencias cardiacas, con el objetivo de construir el atractor en el espacio de fases para cada registro.

Posteriormente, se calcularon los espacios de ocupación de cada atractor y con esta información, a través del método de box-counting, se determinó la dimensión fractal para cada uno de ellos, mediante la superposición de dos rejillas, de 5 y 10 lat/min. A continuación, se calculó la probabilidad de los espacios de ocupación en la rejilla pequeña o $\mathrm{Kp}$ y a partir de este valor se determinó el diagnóstico físico matemático para cada una de las dinámicas. De acuerdo con el estudio previo (en evaluación para publicación), la normalidad se asocia con probabilidades de ocupación espacial iguales o superiores a 0,157 en la rejilla Kp, mientras que la enfermedad aguda se relaciona con valores iguales o inferiores a 0,056.

\section{Análisis estadístico}

Se desenmascararon los diagnósticos convencionales y se tomaron como patrón de oro. Se tomaron medidas de especificidad y sensibilidad, así como de valor predictivo positivo y negativo para comparar la metodología matemática con el diagnóstico clínico establecido.

Dichas medidas se hicieron a través de una clasificación binaria, en la que se observaron verdaderos positivos, verdaderos negativos, falsos positivos y falsos negativos.

Con el objetivo de evaluar la concordancia entre el diagnóstico físico-matemático y el diagnóstico clínico convencional, se calculó el coeficiente kappa a través de la siguiente fórmula:

$K=\frac{C o-C a}{T o-C a}$

\section{Donde:}

Co: número de concordancias observadas, es decir, número de pacientes con el mismo diagnóstico de acuerdo con la nueva metodología propuesta y con el estándar de oro.

To: totalidad de observaciones, es decir, la totalidad de casos normales y patológicos.

Ca: concordancias atribuibles al azar, que se calcularon con base en la siguiente fórmula:

$C a=\left[\left(f_{1} x C_{1}\right) / T o\right]+\left[\left(f_{2} x C_{2}\right) / T o\right]$

Donde $\mathrm{f}_{1}$ es el número de pacientes que presentan valores matemáticos dentro de los límites de normalidad, $C_{1}$ es el número de pacientes diagnosticados clínicamente dentro 
Tabla 1 Diagnóstico convencional de quince de las dinámicas evaluadas

\begin{tabular}{|c|c|c|}
\hline No. & Edad & Diagnóstico convencional \\
\hline 1 & 35 & Normal \\
\hline 2 & 42 & Normal \\
\hline 3 & 34 & Normal \\
\hline 4 & 68 & Normal \\
\hline 5 & 59 & Normal \\
\hline 6 & 45 & $\begin{array}{l}\text { Bradicardia. Disminución moderada } \\
\text { de la variablidad de la frecuencia } \\
\text { cardiaca }\end{array}$ \\
\hline 7 & 37 & $\begin{array}{l}\text { Arritmia. Extrasistolias venticulares } \\
\text { monomórficas aisladas }\end{array}$ \\
\hline 8 & 56 & $\begin{array}{l}\text { Extrasistolia auricular frecuente. } \\
\text { Episodios de trigeminismo }\end{array}$ \\
\hline 9 & 63 & $\begin{array}{l}\text { Arritmia. Extrasistolia ventricular } \\
\text { frecuente con trigeminismo. } \\
\text { Disminución moderada de la } \\
\text { variabilidad de la frecuencia cardiaca }\end{array}$ \\
\hline 10 & 85 & $\begin{array}{l}\text { Extrasistolia ventricular y } \\
\text { supraventricular frecuente }\end{array}$ \\
\hline 11 & 70 & $\begin{array}{l}\text { Ritmo de marcapasos DDD. } \\
\text { Funcionamiento normal }\end{array}$ \\
\hline 12 & 73 & Cardiopatía isquémica FEVI \\
\hline 13 & 59 & $\begin{array}{l}\text { Postoperatorio de ablación. } \\
\text { Bradicardia }\end{array}$ \\
\hline 14 & 79 & IAM. Fracción de eyección del 40\% \\
\hline 15 & 66 & IAMCEST A1 kkl tipo 1. \\
\hline
\end{tabular}

de la normalidad, $f_{2}$ es el número de pacientes que presentan valores matemáticos asociados a enfermedad, $C_{2}$ es el número de pacientes diagnosticados clínicamente con enfermedad y To es el número total de casos normales y con enfermedad.

\section{Aspectos éticos}

Este estudio se enmarca como investigación con riesgo mínimo, de acuerdo con la resolución 8430 de 1993 del Ministerio de Salud de Colombia, pues sólo se realizan cálculos físicos y matemáticos en reportes de exámenes y paraclínicos no invasivos, los cuales ya han sido prescritos. Además, se protegió el anonimato de los pacientes cumpliendo con los principios de la Declaración de Helsinki, proferida por la Asociación Médica Mundial.

\section{Resultados}

En la tabla 1 se observan los diagnósticos convencionales de algunas de las dinámicas del estudio. En la tabla 2 se aprecian los valores de los parámetros encontrados en el estudio; para dinámicas normales los valores de la dimensión fractal oscilaron entre 1,647 y 1,957, mientras que los valores de ocupación espacial para las rejillas $\mathrm{Kp}$ y $\mathrm{Kg}$ estuvieron entre 213 y 426, y 66 y 120, respectivamente. Así mismo, la probabilidad de ocupación en la rejilla Kp arrojó valores entre 0,164 y 0,329 .

Para las dinámicas anormales los valores de la dimensión fractal se encontraron entre 1,404 y 1,953 y los valores de
Tabla 2 Valores de los espacios de ocupación en las rejillas $\mathrm{Kp}$ y $\mathrm{Kg}$ de los atractores, y probabilidad de ocupación espacial del atractor en la rejilla $\mathrm{kp}$

\begin{tabular}{lllll}
\hline Dx & $\mathrm{Kg}$ & $\mathrm{Kp}$ & $\mathrm{D}_{\mathrm{F}}$ & Probabilidad \\
\hline $\mathrm{N}$ & 120 & 426 & 1,83 & 0,329 \\
$\mathrm{~N}$ & 86 & 334 & 1,96 & 0,258 \\
$\mathrm{~N}$ & 76 & 269 & 1,82 & 0,208 \\
$\mathrm{~N}$ & 66 & 221 & 1,74 & 0,171 \\
$\mathrm{~N}$ & 68 & 213 & 1,65 & 0,164 \\
$\mathrm{~A}$ & 52 & 187 & 1,85 & 0,144 \\
$\mathrm{~A}$ & 51 & 174 & 1,77 & 0,134 \\
$\mathrm{~A}$ & 39 & 151 & 1,95 & 0,117 \\
$\mathrm{~A}$ & 34 & 123 & 1,86 & 0,095 \\
$\mathrm{~A}$ & 30 & 106 & 1,82 & 0,082 \\
$\mathrm{~A}$ & 28 & 85 & 1,6 & 0,066 \\
$\mathrm{~A}$ & 23 & 76 & 1,72 & 0,059 \\
$\mathrm{~A}$ & 19 & 52 & 1,45 & 0,04 \\
$\mathrm{~A}$ & 17 & 45 & 1,4 & 0,035 \\
$\mathrm{~A}$ & 13 & 38 & 1,55 & 0,029 \\
\hline
\end{tabular}

Kp: es la rejilla con cuadros pequeños. Kg: rejilla con cuadros grandes. DF: dimensión fractal

ocupación para las rejillas $\mathrm{Kp}$ y $\mathrm{Kg}$ se hallaron entre 38 y 187, y 13 y 52, respectivamente. Así mismo la probabilidad de ocupación en la rejilla Kp arrojó valores entre 0,029 y 0,144 .

Estos valores evidencian que las dimensiones fractales no permiten establecer diferencias entre normalidad y enfermedad, como se había hecho evidente en estudios previos. En contraposición, la probabilidad de ocupación del atractor permite establecer diferencias cuantitativas, confirmando que las dinámicas con enfermedad presentan probabilidades de ocupación menores que las dinámicas normales. De acuerdo con el estudio previo, la normalidad corresponde a probabilidades de ocupación espacial iguales o superiores a 0,157 en la rejilla $\mathrm{Kp}$, mientras que la enfermedad aguda tiene valores iguales o inferiores a 0,056, datos que se confirman con los resultados de esta investigación. Adicionalmente, se observó que dinámicas en estado crónico tiene valores inferiores al límite de normalidad, y que dinámicas en estados agudos, tales como IAM, presentan los valores de probabilidad más pequeños (tabla 2 ).

De este modo se evidenció que los valores de sensibilidad, especificidad, valor predictivo positivo y valor predictivo negativo tuvieron valores de $100 \%$, mientras que el coeficiente kappa tuvo valor de 1.

Los valores anteriores evidencian que las dimensiones fractales no permiten establecer diferencias entre normalidad y enfermedad, como se había hecho evidente en estudios previos. En contraposición, la probabilidad de ocupación del atractor permite establecer diferencias cuantitativas, confirmando que las dinámicas con enfermedad tienen probabilidades de ocupación menores que las dinámicas normales. De acuerdo con el estudio previo, la normalidad corresponde a probabilidades de ocupación espacial iguales o superiores a 0,157 en la rejilla Kp, mientras que la enfermedad aguda presenta valores iguales o inferiores a 0,056 , hecho que también se confirma con los resultados de esta investigación. Además, se observó que 
dinámicas en estado crónico presentan valores inferiores al límite de normalidad y que dinámicas en estados agudos, tales como IAM, tienen los valores de probabilidad más pequeños (tabla 2 ).

De este modo se evidenció que los valores de sensibilidad, especificidad, valor predictivo positivo y valor predictivo negativo tuvieron valores de $100 \%$, mientras que el coeficiente kappa tuvo valor de 1.

\section{Discusión}

Este es el primer estudio en el cual se confirma la utilidad clínica y diagnóstica de la metodología fundamentada en los sistemas dinámicos y la teoría de la probabilidad, desarrollada para la evaluación de las dinámicas cardiacas, alcanzando los máximos valores permitidos de especificidad y sensibilidad. Por su objetividad y reproducibilidad, esta metodología puede aplicarse a cualquier caso, ya sea proveniente de estudios Holter o de registros electrocardiográficos continuos de $\mathrm{UCl}$, independiente del tipo de enfermedad, intervenciones, edad $u$ otro factor de riesgo, en individuos mayores de veintiún años.

Este método es de suma utilidad en la diferenciación entre normalidad y enfermedad aguda, pues además establece un rango de valores característicos de evolución entre ambos estados. La metodología se configura como una forma de evaluación de los registros electrocardiográficos que se basa en conceptos matemáticos de fácil aplicación, siendo útil para evaluar la eficiencia de procesos quirúrgicos o terapia farmacológica e igualmente para realizar un seguimiento más objetivo de pacientes en la UCl.

En un estudio prospectivo desarrollado en pacientes con alto riesgo de muerte cardiaca súbita, se propuso como hipótesis que la evaluación de las variaciones en la morfología de la onda $T$ en respuesta a las variaciones en el intervalo RR, podría utilizarse como un marcador de heterogeneidad espacial de la restitución, y que adicionalmente esta relación se asocia con mayor vulnerabilidad a las arritmias ventriculares ${ }^{17}$. El estudio encontró un índice que cuantifica el cambio morfológico de la onda $\mathrm{T}$ por incremento de RR y, por tanto, representa la restitución de la morfología de la onda T (RMT), es decir, el índice cuantifica las variaciones morfológicas de la onda $T$ y se considera como la mejor estimación de la heterogeneidad de la restitución de la repolarización que los marcadores basados en intervalos. Sin embargo, esta hipótesis necesita estudios futuros para evaluar la relación entre RMT y otros índices de restitución, como el índice de intensidad de restitución regional (R212) recientemente propuesto como marcador de riesgo de muerte subita ${ }^{18}$.

Por su parte, esta metodología puede sintetizar la cantidad de variables implícitas en los anteriores estudios, los cuales requieren de gran número de procedimientos para seleccionar y medir la onda T. En cuanto a los marcadores de riesgo de muerte súbita definidos en el momento en que está sucediendo el episodio, con la idea de que es este el momento de mayor información, la investigación puede requerir de mayor número de sujetos para definir el índice más apropiado y aplicable para todos los casos. Así mismo, esta metodología muestra la posibilidad de hacer un análisis más profundo a partir de los valores de la frecuencia cardiaca máxima, mínima y total de latido por hora, representados en un atractor cardiaco, cuyos espacios de ocupación del atractor indican en el tiempo, la evolución a un estado de agudización de la dinámica cardiaca sin esperar que acontezca el evento adverso.

Este trabajo, además, presenta un avance importante respecto al trabajo en el que se planteó originalmente el método diagnóstico, pues mientras que en dicha investigación se evaluaron dinámicas en estado normal y agudo, en esta se incluyeron dinámicas en estados crónicos, demostrándose que en todos los casos estas dinámicas tienen probabilidades de ocupación espacial inferiores al estado de normalidad, y que dicho valor de probabilidad disminuye a medida que se se dan dinámicas en estados más agudos. Así, por ejemplo, la dinámica 6 de la tabla 1 presentó bradicardia y disminución moderada de la VFC, que es una afección leve en comparación con la dinámica 14 , que presentó IAM y una fracción de eyección del 40\%. En el primer caso se encontró una probabilidad de ocupación del atractor de 0,144 , mientras que en el segundo la probabilidad fue de solo 0,035 , más de diez veces menor.

En Cardiología se había estudiado la variabilidad de la frecuencia cardiaca ${ }^{19}$, con algunas limitaciones en su aplicabilidad; sin embargo, han surgido métodos cuantitativos que evidencian una autoorganización del sistema, desde la perspectiva físico-matemática como el que se empleó en este trabajo.

Esta perspectiva ha permitido el advenimiento de una ley matemática exponencial para la evaluación de la dinámica cardiaca, cuya aplicabilidad clínica, ha sido confirmada en pacientes con arritmia $^{20}$. El concepto de ocupación espacial de los atractores, que fundamenta dicha ley, también ha sido útil para evaluar la dinámica cardiaca neonatal y detectar alteraciones previas de sepsis ${ }^{21}$. Desde la probabilidad, se han observado diferencias entre normalidad y enfermedad y se han identificado alteraciones leves subdiagnosticadas de forma convencional, en pacientes con arritmias y marcapasos ${ }^{20,22}$. También, se desarrolló una metodología que permite hacer diagnósticos precisos de los sistemas cardiacos a partir de la proporción de la entropía de atractores ${ }^{23}$. Al ser aplicada en pacientes de la unidad de cuidados coronarios, se mostró que procesos de agudización que no son de fácil seguimiento con métodos usuales, quedan expuestos y adecuadamente diagnosticados a través de este método ${ }^{24}$. En la misma línea, esta investigación reafirma la relevancia de la aplicación de metodologías basadas en el pensamiento de las ciencias básicas, como la Física y las Matemáticas, pues su carácter objetivo da luces a la distinción acertada de estados de enfermedad, con lo cual se convierten en herramientas de suma utilidad clínica, al dejar de lado factores de riesgo y consideraciones estadísticas o poblacionales por su carácter acausal y el método inductivo subyacente ${ }^{25}$, procedente de la física teórica moderna.

Esta forma de proceder ha permitido realizar contribuciones de carácter predictivo y diagnóstico en áreas como la Inmunología 26 , la Infectología ${ }^{27}$ y la Salud pública (en temas como la malaria) ${ }^{28}$; también se han desarrollado caracterizaciones y diagnósticos a nivel celular y arterial $^{29-31}$ y se ha logrado predecir mortalidad en la $\mathrm{UCl}^{32}$. 


\section{Financiación}

Producto derivado del proyecto INV-CIAS- 2047, financiado por la Vicerrectoría de Investigaciones de la Universidad Militar Nueva Granada-Vigencia 2016, y de la Línea de Profundización, Internado Especial y Semillero "Teorías Físicas y Matemáticas Aplicadas a la Medicina”.

\section{Conflictos de interés}

Ninguno.

\section{Agradecimientos}

A la Universidad Militar Nueva Granada, por su apoyo a nuestros procesos investigativos, especialmente al Fondo de Investigaciones de la Universidad, a la Vicerrectoría de Investigaciones y la Facultad de Ciencias Básicas y Aplicadas.

A la Doctora Yanneth Méndez, Vicerrectora académica, a la Ingeniera Marcela Iregui, Vicerrectora de Investigaciones, al Doctor Juan José Filgueira, Director del Centro de Investigaciones de la Facultad de Ciencias Básicas y Aplicadas y al Doctor Carlos Andrés Coy, Decano de la Facultad de Ciencias Básicas y Aplicadas.

Al Centro de Investigaciones de la Clínica del Country, a la Doctora Adriana Lizbeth Ortiz, epidemióloga, a la enfermera jefe Silvia Ortiz, y a los Doctores Tito Tulio Roa, Director de Educación Médica, Jorge Alberto Ospina, Director Médico, y Alfonso Correa, Director del Centro de Investigaciones, por el apoyo a nuestro grupo de investigación.

\section{Bibliografía}

1. Devaney R. A first course in chaotic dynamical systems theory and experiments. Reading Mass: Addison-Wesley; 1992.

2. Peitgen H. Strange attractors the locus of chaos. En: Chaos, Fractals, editores. New Frontiers of Science. New York: Springer-Verlag; 1992. p. 655-768.

3. Feynman RP, Leighton RB, Sands M, Probabilidad, En: Feynman RP, Leighton RB, Sands M. Física, 1. Wilmington: Addison-Wesley Iberoamericana, S. A; 1964. p. 6-11, 6-16.

4. Gough N. Fractals, chaos, and fetal heart rate. Lancet. 1992;339:182-3.

5. Denton T, Diamond G, Helfant RH, Khan S, Karagueuzian H, et al. Fascinating rhythm: a primer on chaos theory and its application to cardiology. Am Heart J. 1990;6:1419-40.

6. Rodríguez J. Mathematical law of chaotic cardiac dynamic: Predictions of clinic application. Journal of Medicine and Medical Science. 2011;2:1050-9.

7. Pineda M, Matiz H, Rozo R. Enfermedad coronaria. Bogotá: Editorial Kimpres Ltda; 2002.

8. OMS-Centro de prensa. Enfermedades Cardiovasculares. Nota informativa [Acceso Ene 2011]. Disponible en: URL: http://www.who.int/mediacentre/factsheets/fs317/es/index. html.

9. Rodríguez J, Prieto S, Ortiz L, Bautista A, Bernal P, Avilán N. Diagnóstico matemático de la monitoría fetal aplicando la ley de Zipf-Mandelbrot. Revista de la Facultad de Medicina de la Universidad Nacional de Colombia. 2006;54:96-107.

10. Gallo J, Farbiarz J, Álvarez D. Análisis espectral de la variabilidad de la frecuencia cardíaca. IATREIA. 1999;12:61-71.
11. Goldberger A, Amaral L, Hausdorff JM, Ivanov P, Peng Ch, Stanley HE. Fractal dynamics in physiology: alterations with disease and aging. Proceedings of the National Academy of Sciences. 2002;99:2466-72.

12. Voss A, Schulz S, Schroeder R, Baumert M, Caminal P. Methods derived from nonlinear dynamics for analysing heart rate variability. Philosophical Transactions of Royal Society A. 2009;367:277-96.

13. Huikuri HV, Mäkikallio TH, Peng Ch, Goldberger AL, Hintze $\mathrm{U}$, Moller M. Fractal correlation properties of R-R interval dynamics and mortality in patients with depressed left ventricular function after an acute myocardial infartion. Circulation. 2000;101:47-53.

14. Rodríguez J, Prieto S, Bernal P, Soracipa Y, Salazar G, Isaza D, et al. Nueva metodología de ayuda diagnóstica de la dinámica geométrica cardiaca: dinámica cardiaca caótica del Holter. Revista de la Academia Colombiana de Ciencias. 2011;35: $5-12$.

15. Rodríguez J, Prieto S, Correa C, Oliveros H, Soracipa Y, Amaya J, et al. Sistemas dinámicos aplicados a la disminución del tiempo de diagnóstico de la dinámica cardiaca de 24 a 16 horas en Holter y registros electrocardiográficos continuos. X Congreso de Medicina Crítica y Cuidado Intensivo. Colombia: Cartagena; 2015.

16. Rodríguez J, Correa C, Prieto S, Bernal P, Forero G, Salazar G, et al. Confirmación del método de ayuda diagnóstica de la dinámica cardiaca de aplicación clínica desarrollado con base en la teoría de la probabilidad. Revista de la Facultad de Medicina. 2011;19:167-77.

17. Kasahara K, Shiobara M, Nakamura S, Yamashiro K, Yana K, Ono T. Sudden cardiac arrest risk stratification based on 24-hour Holter ECG statistics. Send to Conf Proc IEEE Eng Med Biol Soc. 2015;2015:5817-20.

18. Nicolson WB, McCann GP, Brown PD, Sandilands AJ, Stafford PJ, Schlindwein FS, et al. A novel surface electrocardiogram-based marker of ventricular arrhythmia risk in patients with ischemic cardiomyopathy. J Am Heart Assoc. 2012;1:e001552.

19. Chen WL, Kuo CD. Characteristics of heart rate variability can predict impending septic shock in emergency department patients with sepsis. Acad Emerg Med. 2007;14:3920-7.

20. Rodríguez J, Narváez R, Prieto S, Correa C, Bernal P, Aguirre $\mathrm{G}$, et al. The mathematical law of chaotic dynamics applied to cardiac arrhythmias. J Med Med Sci. 2013;4:291-300.

21. Rodríguez J, Prieto S, Flórez M, Alarcón C, López R, Aguirre G, et al. Physical- mathematical diagnosis of cardiac dynamic on neonatal sepsis: predictions of clinical application. J Med Med Sci. 2014;5:102-8.

22. Rodríguez J, Prieto S, Correa C, Bernal P, Vitery S, Álvarez $\mathrm{L}$, et al. Diagnóstico cardiaco basado en la probabilidad aplicado a pacientes con marcapasos. Acta Médica Colombiana. 2012;37:183-91

23. Rodríguez J, Prieto S, Domínguez D, Melo M, Mendoza F, Correa C, et al. Mathematical-physical prediction of cardiac dynamics using the proportional entropy of dynamic systems. J. Med. Med. Sci. 2013;4:370-81.

24. Rodríguez J, Prieto S, Melo M, Domínguez D, Correa C, Soracipa Y, et al. Entropía proporcional de la dinámica cardiaca aplicada al diagnóstico de pacientes de la Unidad de Cuidados Intensivos. Med (Bogotá). 2013;35:17-28.

25. Feynman R, Leighton RB, Sands M, Comportamiento cuántico, En: Feynman R, Leighton RB, Sands M. Física, 1. Wilmington: Addison-Wesley Iberoamericana S.A; 1987. p. 1-37.

26. Rodríguez J, Bernal P, Prieto P, Correa C, Álvarez L, Pinilla L, et al. Predicción de unión de péptidos de Plasmodium falciparum al HLA clase II Probabilidad, combinatoria y entropía aplicadas a las proteínas MSP-5 y MSP-6. Archivos de Alergia e Inmunología Clínica. 2013;44:7-14. 
27. Rodríguez J, Prieto S, Correa C, Mora J, Bravo J, Soracipa Y, et al. Predictions of CD4 lymphocytes' count in HIV patients from complete blood count BMC Medical Physics. BMC Medical Physics. 2013;13:3.

28. Rodríguez J. Método para la predicción de la dinámica temporal de la malaria en los municipios de Colombia. Rev Panam Salud Pública. 2010;27:211-8.

29. Rodríguez J, Prieto S, Correa C, Bernal P, Puerta G, Vitery $S$, et al. Theoretical generalization of normal and sick coronary arteries with fractal dimensions and the arterial intrinsic mathematical harmony. BMC Med Phys. 2010;10: $1-6$.
30. Prieto S, Rodríguez J, Correa C, Soracipa Y. Diagnosis of cervical cells based on fractal and Euclidian geometrical measurements: Intrinsic Geometric Cellular Organization. BMC Medical Physics. 2014;14:1-9.

31. Correa C, Rodríguez J, Prieto S, Álvarez L, Ospino B, Munevar A, et al. Geometric diagnosis of erythrocyte morphophysiology: Geometric diagnosis of erythrocyte. J. Med. Med. Sci. 2012;3:715-20.

32. Rodríguez J. Dynamical systems applied to dynamic variables of patients from the Intensive Care Unit (ICU). Physical and mathematical Mortality predictions on ICU. J. Med. Med. Sci. 2015;6:102-8. 